\title{
Laparoscopic adjustable gastric banding. A prospective randomized study comparing the Swedish Adjustable Gastric Band and the MiniMizer Extra: one-year results
}

\author{
Tomas Abalikšta ${ }^{1,2}$, Gintautas Brimas ${ }^{1,2}$, Kęstutis Strupas ${ }^{1,2}$ \\ 1Vilnius University Medical Faculty, Vilnius, Lithuania \\ 2Vilnius University Hospital Santariškų Klinikos, Vilnius, Lithuania
}

Videosurgery and Other Miniinvasive Techniques 2011; 6 (4): 207-216 DOI: 10.5114/wiitm.2011.26254

\begin{abstract}
Introduction: A number of different adjustable gastric bands are available for laparoscopic adjustable gastric banding (LAGB). Few attempts have been made to compare the influence of band design differences for efficiency and complication rate and conflicting results have emerged from comparative studies.

Aim: To compare SAGB (Swedish Adjustable Gastric Band) and MiniMizer Extra adjustable gastric bands.

Material and methods: One hundred and three patients were included in the prospective randomized study. All patients underwent LAGB. The SAGB was used in 49 and MiniMizer Extra in 54 patients. The primary endpoint was weight loss, and secondary endpoints were complication rate, correction of co-morbidities and improvement of quality of life.

Results: There were no early complications. A significant difference in the proportion of patients who have reached good or excellent weight loss results ( $\geq 50 \%$ of initial excess body mass index loss) was found in favour of the MiniMizer Extra group (29.6\% vs. 8.2\%, $p=0.006$ ). No difference was found in other weight loss parameters, resolution of co-morbidities and improvement of quality of life. One oesophageal dilatation and one leakage were diagnosed in the MiniMizer Extra group. Five band penetrations (9.3\%) were diagnosed in the MiniMizer Extra group and no penetrations in the SAGB group ( $p=0.069)$.

Conclusions: No major significant differences were found between the compared bands. Further results need to be confirmed by longer follow-up.
\end{abstract}

Key words: laparoscopic adjustable gastric banding, morbid obesity, bariatric surgery.

\section{Introduction}

Laparoscopic adjustable gastric banding (LAGB) is one of the methods for surgical treatment of morbid obesity (body mass index $(\mathrm{BMI})>40 \mathrm{~kg} / \mathrm{m}^{2}$ or $>35$ $\mathrm{kg} / \mathrm{m}^{2}$ with obesity-associated co-morbidities). This method, introduced by Kuzmak [1] in 1985 and Hallberg and Forsell, has been performed laparoscopically since 1993 [3]. It is estimated that LAGB represents about $42 \%$ of bariatric operations performed worldwide [4]. There are a number of different adjustable gastric bands available on the international market for performing LAGB. These bands differ in size, filling volume [5], internal pressure (and possibly pressure on the gastric wall), fixation method and materials they are made of. Few attempts have been made to compare the influence of these band design differ- 
ences for efficiency and complication rate and conflicting results have emerged from comparative studies that have significant shortcomings: usually nonrandomised or retrospective small studies with short follow-up [6-17].

\section{Aim}

The aim of our study was to compare two adjustable gastric bands - SAGB (Swedish Adjustable Gastric Band; Obtech Medical, Switzerland) and MiniMizer Extra (Bariatric Solutions $\mathrm{GmbH}$, Switzerland) in terms of weight loss, complication rate, correction of co-morbidities and improvement of quality of life. These two bands were chosen for the most possible differences in their design.

\section{Material and methods}

The study protocol was approved by the Lithuanian Bioethics Committee on November 6, 2008. Patients aged $18-70$ years with a BMI more than 40 $\mathrm{kg} / \mathrm{m}^{2}$ and patients with BMI between $35 \mathrm{~kg} / \mathrm{m}^{2}$ and $40 \mathrm{~kg} / \mathrm{m}^{2}$ with associated co-morbidities were considered eligible for LAGB. Exclusion criteria were contraindications for laparoscopy, previous bariatric surgery, pregnancy and patient's refusal. Signed informed consent was obtained from all patients. Patients were randomly assigned to groups by choosing from two identical sealed envelopes with the different band name inside. All operations were performed by a single surgeon with prior experience of more than 400 LAGB operations using SAGB and MiniMizer Extra bands.

\section{Preoperative data}

Extensive preoperative evaluation was done by a multidisciplinary team, consisting of an endocrinologist, gastroenterologist, dietician, cardiologist and bariatric surgeon. Broad laboratory blood tests, upper gastrointestinal tract endoscopy, abdominal and cardiac sonoscopy and upper gastrointestinal tract radiography were performed on all included patients according to the study protocol. Initial body weight (IBW) was measured and body composition analysis (hand-to-foot bioelectrical impedance analysis) for initial body fat mass (IFM) and percent of initial fat mass (\%IFM) evaluation was performed. Initial BMI (IBMI) and initial fat mass index (IFMI = IFM/(height in metres) ${ }^{2}, \mathrm{~kg} / \mathrm{m}^{2}$ ) were calculated from these data. Fat mass evaluation was possible only if \%IFM did not exceed $50 \%$ (technical limitation of the bioelectrical impedance analysis device); thus all patients with \%IFM more than 50\% were involved in statistical analysis as if they had $50 \%$ of \%IFM. Initial excess body weight (IEW) was calculated using the formula $\mathrm{IEW}=$ (preoperative body weight) - (upper limit of normal body weight). Upper limit of normal body weight is body weight if patients' BMI were $25 \mathrm{~kg} / \mathrm{m}^{2}$ and is calculated by the following formula: upper limit of normal body weight $=$ (height in metres $)^{2} \times 25$. Initial excess body mass index (IEBMI) was calculated by taking $25 \mathrm{~kg} / \mathrm{m}^{2}$ from IBMI. Metabolic syndrome was diagnosed by Adult Treatment Panel III Guidelines [18]. Quality of life (QoL) was evaluated by the MooreheadArdelt Quality of Life Questionnaire II (M-A QoLQII) in points from -3 to 3 [18-20]. According to the $M-A$ QoLQII scoring key the QoL is stated as very poor from -3 to -2.1 points, poor from -2 to -1 point, fair from -1 to 1 point, good from 1.1 to 2 points and very good from 2.1 to 3 points. Thromboembolic prophylaxis was provided using low-molecular-weight heparin(nadroparin) $12 \mathrm{~h}$ before the operation and on the first postoperative day until mobilization was achieved. Furthermore, mechanical calf compression was also used for the prevention of deep venous thrombosis during surgery. One dose of $2 \mathrm{~g}$ cefuroxime was administered intravenously 10-15 min before the operation for infection prophylaxis. All patients underwent general anaesthesia and nasogastric suction.

\section{Surgical technique}

All patients underwent LAGB. Two different adjustable gastric bands were used: the SAGB and the MiniMizer Extra (Figure I). Pars flaccida technique was used for both bands [21]. Fixation of the band on the anterior gastric wall was achieved by creating a gastric fold over the band with four non-absorbable gastro-gastric sutures in the case of SAGB (fixation with plication) or directly suturing the fixation loops of the MiniMizer Extra band to the stomach wall (fixation without plication) [22]. The band was left empty at the end of the procedure. The MiniMizer Extra band was closed either in the first (wider) or second (tight) position according to the stomach diameter inside the band. The SAGB-Velocity Port and Applier were used in the case of the SAGB band and MiniMizer Port Medium was used in the case of the MiniMizer Extra band. 

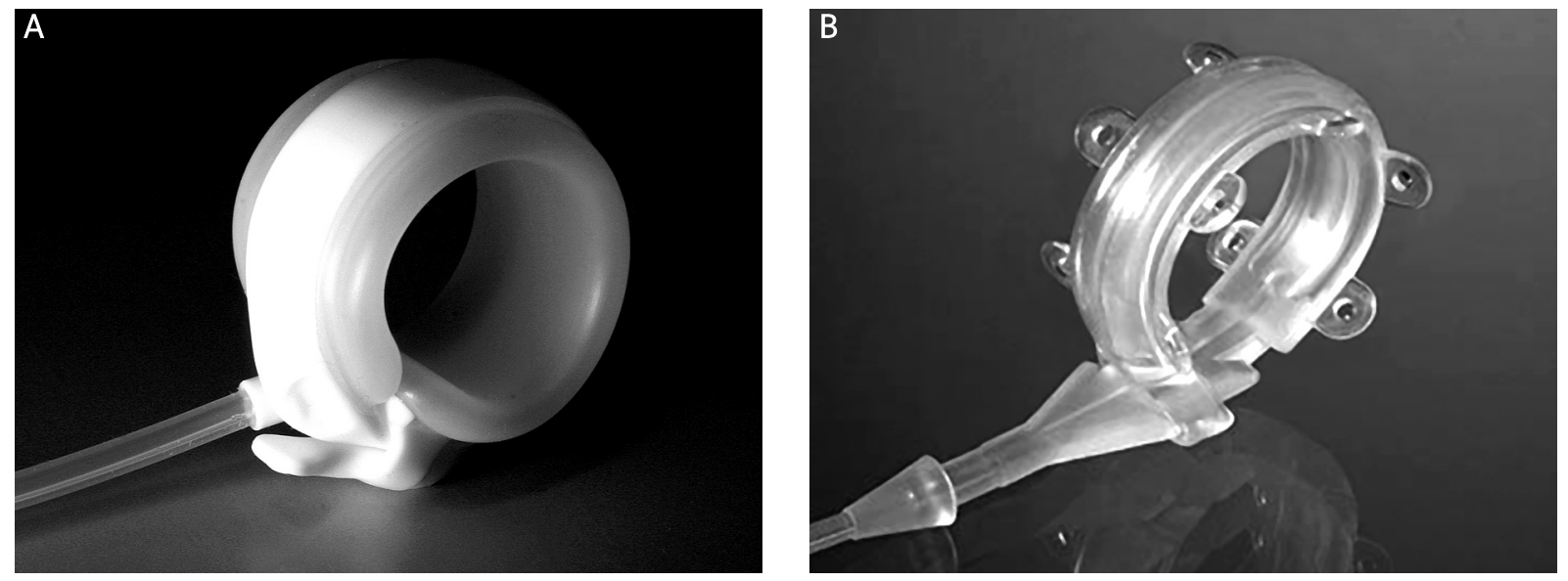

Figure 1. Compared adjustable gastric bands. A - SAGB, B - MiniMizer Extra

\section{Postoperative care}

Early mobilization occurred 3-4 h after the operation. The next day after surgery patients could drink liquids. Pain and other unpleasant symptoms, such as nausea, discomfort in the epigastric region, shoulder pain, tachycardia and others, were evaluated with a special questionnaire by a visual analogue scale (VAS100). Patients with normal conditions were discharged on the second postoperative day after being instructed to follow a semi-liquid diet for the first postoperative month. Patients were also provided with written diet instructions.

\section{Band adjustment}

Band adjustment rate was not influenced by the study protocol and was performed according to the accepted standards of our clinic. The bands were left empty in the widest possible position at the end of the operation. The patients were asked to arrive for band adjustment in the case of ceasing to lose weight for more than 2 weeks, but not earlier than four weeks after the operation. All other adjustments were also performed in case of ceasing to lose weight for more than two weeks, but not earlier than 4 weeks after previous adjustment. The access to the port was detected sonoscopically. The average filling volume of SAGB was $2.5 \mathrm{ml}$ (not more than $4 \mathrm{ml}$ ) of saline solution for the first adjustment, $1.5 \mathrm{ml}$ for the second adjustment, $1 \mathrm{ml}$ for the third adjustment and $0.5 \mathrm{ml}$ for all later adjustments. The average filling volume of the MiniMizer Extra band was $1.2 \mathrm{ml}$ (not more than $2 \mathrm{ml}$ ) of saline solution for the first adjustment, $0.6 \mathrm{ml}$ for the second adjustment and $0.3 \mathrm{ml}$ for all later adjustments. The degree of restriction was usually controlled by clinical signs only (patient's ability to drink liquids). Upper gastrointestinal tract radiography was performed in cases when two consecutive band adjustments were unsuccessful to reach sufficient restriction or if band leak or band overfilling was suspected.

\section{Follow-up and evaluation}

Patients were evaluated one year after the operation by the same multidisciplinary team. The same laboratory blood tests, upper gastrointestinal tract endoscopy, cardiac sonoscopy, upper gastrointestinal tract radiography, body composition analysis and the evaluation of QoL were repeated. The primary endpoints were percentage of initial excess weight loss (\%IEWL), percentage of initial excess body mass index loss (\%IEBMIL), percentage of initial body fat mass loss (\%IFML) and percentage of initial fat mass index loss (\%IFMIL) [23]. Secondary endpoints were early and late complications, reoperations, the proportion of patients with \%IEWL of at least $50 \%$, changes in quality of life and co-morbidities. Early complications were complications arising during the first postoperative month. Major early complications were life-threatening and/or led to reoperation. Major late complications were life-threatening and/or led to band removal. Patients whose band had to be removed were excluded from further 
weight loss analysis; however, they remained included in the analysis regarding the percentage of patients achieving $50 \%$ of $\%$ IEWL, in which they were considered as failures. Final evaluation of the results was done by the Bariatric Analysis and Reporting Outcome System (BAROS) [19, 20, 24]. Outcome groups by the BAROS scoring key are as follows: for patients with co-morbidities, failure in case of 1 point or less, fair in case of $>1$ to 3 points, good in case of $>3$ to 5 points, very good in case of $>5$ to 7 points and excellent in case of $>7$ to 9 points; for patients without co-morbidities, failure in case of 0 points or less, fair in case of $>0$ to 1.5 points, good in case of $>1.5$ to 3 points, very good in case of $>3$ to 4.5 points and excellent in case of $>4.5$ to 6 points. Only four co-morbidities (hypertension, cardiovascular diseases, dyslipidaemia and type II diabetes) from the BAROS major co-morbidities list were included in BAROS evaluation. We did not include changes in sleep apnoea, osteoarthritis and infertility, because there was no possibility to perform formal sleep study, osteoarthritis imaging evaluation and infertility/hormonal studies as required by BAROS for diagnosis of these conditions.

\section{Statistical analysis}

All data analysis was performed using the software program SPSS Statistics 17.0. Descriptive statistics were mean \pm SD and range for parametric continuous variables (after confirmation of normal distribution with one-sample Kolmogorov-Smirnov test) and frequencies for categorical variables. Student's $t$ test or Mann-Whitney $U$ test for continuous variables and $\chi^{2}$ or Fisher exact test for categorical variables were used to analyse differences between groups. Differences were considered significant if $p<0.05$.

\section{Results}

\section{Preoperative data}

A total of 103 patients were included in this study between 1 January 2009 and 31 January 2010. There were 69 women (67\%) and 34 men (33\%). Patients' mean age was $46.1 \pm 11.5$ years and weight was 141.9 $\pm 24.2 \mathrm{~kg}$. The SAGB was used in 49 patients and MiniMizer Extra in 54 patients. All patients had co-morbidities and most of them had multiple co-morbidities (5.2 \pm 2.2 (range 1-11) co-morbidities per patient).
Thirty-three patients (32\%) had type II diabetes mellitus, 45 (43.7\%) gastric disorders (erosions, hiatal hernias or GERD), 82 (79.6\%) arterial hypertension, $29(28.2 \%)$ respiratory diseases, 28 (27.2\%) sleep apnoea, 21 (20.4\%) cardiac pathology, 19 (18.4\%) genital disorders, 1 (1\%) liver disease, 28 (27\%) gall bladder stones, 71 (68.9\%) knee pain, 80 (77.7\%) spinal pain, 34 (33\%) varicose veins of the leg, $6(5.8 \%)$ elephantiasis, $11(10.7 \%)$ psoriasis, 24 (23.3\%) thyroid gland diseases, 9 (8.7\%) urinary tract diseases, 13 (12.6\%) depression and 6 (5.8\%) hernias. Metabolic syndrome was diagnosed in $79(77.5 \%)$ patients. Initial QoL was $0.02 \pm 1.2$ points by M-A QoLQII. The preoperative characteristics of the patients, initial frequency of co-morbidities and QoL did not differ between groups. The preoperative characteristics are listed in Table I and shown in Figures 2 and 3.

\section{Operation}

The operative time was $64.6 \pm 18.7 \mathrm{~min}$. In 8 cases additional procedures were performed during the operation: 4 small previously undiagnosed formations were removed from the anterior gastric wall (2 gastrointestinal stroma tumour (GIST), 1 fibroadenoma and 1 ectopic pancreatic tissue formation), extensive dissection of massive adhesions after previous operations was performed in 2 cases, diaphragmatic crura were sutured to repair large hiatal hernia in 1 case and cystectomy of the ovarian cyst was performed in 1 case. We encountered five minor intraoperative complications: gastric lesion in one case, bleeding from a gastric vessel in one case, hepatic lesions caused by hepatic retractor in 2 cases and loss of the needle in 1 case. All complications were diagnosed and solved during the same operation. The time of these additional procedures was excluded from the whole operative time for comparative reasons, because this additional time was not associated with either of the bands. Operative time, duration of pneumoperitoneum (i.e. band placement and fixation time) and injection port implantation time are shown in Table II. Pain level and the rate of other unpleasant symptoms do not differ between groups.

\section{Band adjustment}

The bands were adjusted $2.7 \pm 2.2$ times (range 0 -10) in the SAGB group and $2.4 \pm 1.2$ times (range $0-5)$ in the MiniMizer Extra group ( $p=0.514)$. 
Table I. Preoperative characteristics of patients

\begin{tabular}{|c|c|c|c|c|}
\hline \multirow[t]{2}{*}{ Variable } & \multirow[t]{2}{*}{ All patients } & \multicolumn{2}{|c|}{ Comparative groups } & \multirow{2}{*}{$\begin{array}{c}\text { Difference } \\
\text { between } \\
\text { groups, } p\end{array}$} \\
\hline & & SAGB & MiniMizer Extra & \\
\hline Number of patients & 103 & 49 & 54 & 0.622 \\
\hline Females/males, $n(\%)$ & $\begin{array}{l}69 / 34 \\
(67 / 33)\end{array}$ & $\begin{array}{c}31 / 18 \\
(63.3 / 36.7)\end{array}$ & $\begin{array}{c}38 / 16 \\
(70.4 / 29.6)\end{array}$ & 0.530 \\
\hline Age [years] & $\begin{array}{c}45.9 \pm 11.7 \\
(21-70)\end{array}$ & $\begin{array}{c}46.1 \pm 11.5 \\
(21-67)\end{array}$ & $\begin{array}{c}45.8 \pm 11.9 \\
(22-70)\end{array}$ & 0.869 \\
\hline IBW [kg] & $\begin{array}{l}137.6 \pm 24.4 \\
(87.4-199.3)\end{array}$ & $\begin{array}{l}141.9 \pm 24.2 \\
(87.4-199.3)\end{array}$ & $\begin{array}{c}133.8 \pm 24.1 \\
(88.5-196.5)\end{array}$ & 0.093 \\
\hline IFM [kg] & $\begin{array}{l}60.4 \pm 12.9 \\
(33.4-95.5)\end{array}$ & $\begin{array}{c}61.5 \pm 13.3 \\
(37.3-95.5)\end{array}$ & $\begin{array}{c}59.4 \pm 12.7 \\
(33.4-85)\end{array}$ & 0.417 \\
\hline$\% I F M[\%]$ & $\begin{array}{c}43.9 \pm 5.5 \\
(30-50)\end{array}$ & $\begin{array}{c}43.3 \pm 5.7 \\
(31-50)\end{array}$ & $\begin{array}{c}44.5 \pm 5.3 \\
(30-50)\end{array}$ & 0.273 \\
\hline IBMI [kg/m²] & $\begin{array}{l}47.5 \pm 7.3 \\
(35-68.3)\end{array}$ & $\begin{array}{l}48.6 \pm 7.9 \\
(35-68.3)\end{array}$ & $\begin{array}{l}46.5 \pm 6.7 \\
(35-62.4)\end{array}$ & 0.157 \\
\hline IFMI [kg/m²] & $\begin{array}{c}21.1 \pm 4.9 \\
(11.8-34.1)\end{array}$ & $\begin{array}{c}21.3 \pm 5.3 \\
(13.1-34.1)\end{array}$ & $\begin{array}{l}20.9 \pm 4.6 \\
(11.8-31.2)\end{array}$ & 0.703 \\
\hline IEW [kg] & $\begin{array}{c}64.9 \pm 21.2 \\
(24.9-116.5)\end{array}$ & $\begin{array}{c}68.5 \pm 22.1 \\
(24.9-116.5)\end{array}$ & $\begin{array}{c}61.8 \pm 20.0 \\
(25.3-114.6)\end{array}$ & 0.107 \\
\hline IEBMI [kg/m²] & $\begin{array}{l}22.5 \pm 7.4 \\
(10-43.3)\end{array}$ & $\begin{array}{l}23.6 \pm 7.9 \\
(10-43.3)\end{array}$ & $\begin{array}{l}21.5 \pm 6.7 \\
(10-37.4)\end{array}$ & 0.157 \\
\hline
\end{tabular}

IBW - initial body weight, IFM - initial fat mass, \%IFM - percentage of initial fat mass, IBMI - initial body mass index, IFMI - initial fat mass index, IEW - initial excess weight, IEBMI - initial excess body mass index. Data are given as mean $\pm S D$ (range) unless otherwise indicated

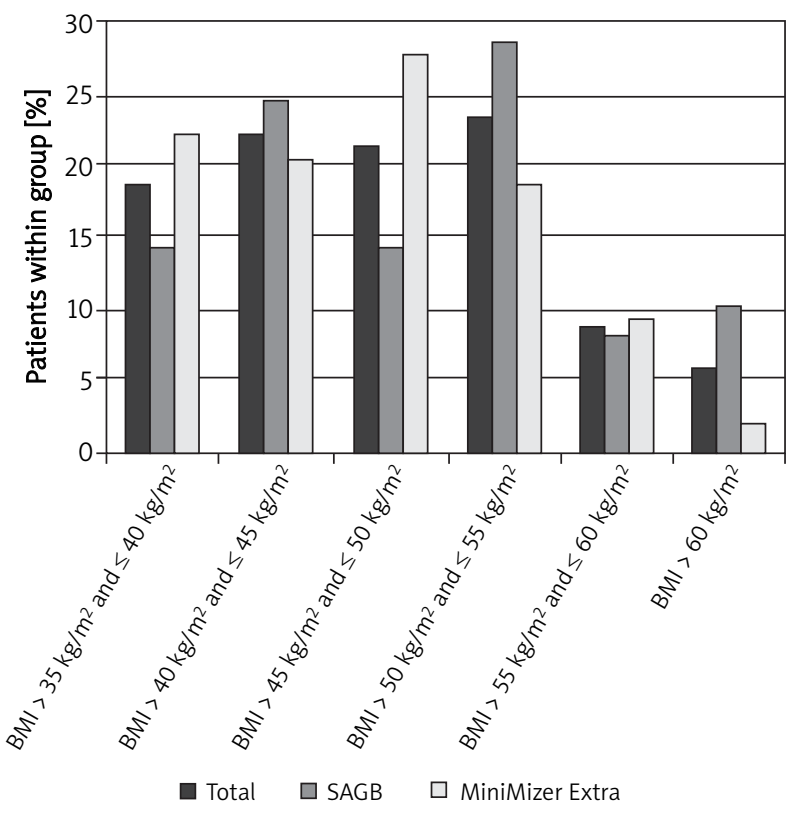

Figure 2. Distribution of patients by preoperative BMI

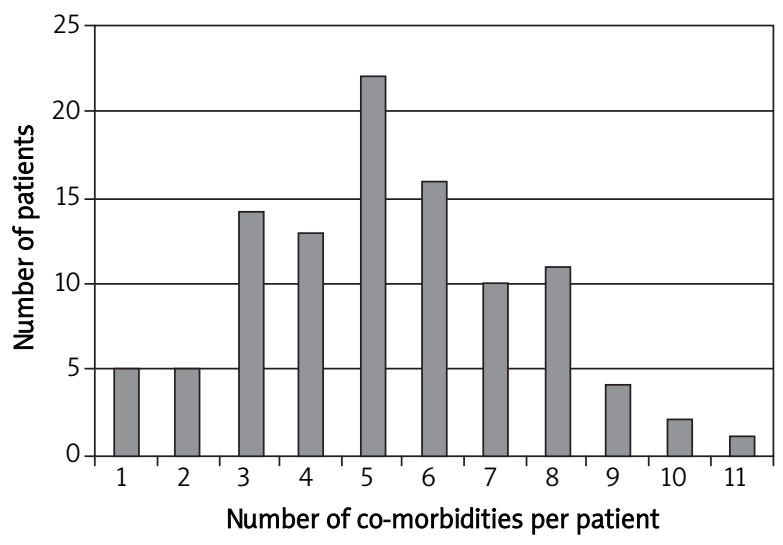

Figure 3. Distribution of patients by the number of co-morbidities 
Table II. Operative times

\begin{tabular}{|lcccc|}
\hline Variable & All patients & \multicolumn{2}{c}{ Comparative groups } & $\begin{array}{c}\text { Difference } \\
\text { between } \\
\text { groups, } p\end{array}$ \\
\cline { 3 - 5 } & & SAGB & MiniMizer Extra & 0.110 \\
\hline Operative time [min] & $\begin{array}{c}64.6 \pm 18.7 \\
(40-160)\end{array}$ & $\begin{array}{c}67.7 \pm 21.9 \\
(40-160)\end{array}$ & $\begin{array}{c}61.8 \pm 14.8 \\
(45-120)\end{array}$ & 0.016 \\
\hline Duration of pneumoperitoneum & $\begin{array}{c}51.6 \pm 18.5 \\
(30-150)\end{array}$ & $\begin{array}{c}56.2 \pm 21.5 \\
(30-150)\end{array}$ & $\begin{array}{c}47.3 \pm 14.1 \\
(30-105)\end{array}$ & $<0.001$ \\
\hline [min] & $\begin{array}{c}13.0 \pm 2.9 \\
(10-20)\end{array}$ & $\begin{array}{c}11.4 \pm 2.5 \\
(10-20)\end{array}$ & $\begin{array}{c}14.4 \pm 2.5 \\
(10-20)\end{array}$ & \\
\hline Injection port implantation time & & & \\
[min] & &
\end{tabular}

Data are given as mean $\pm S D$ (range)

\section{Early complications and lost follow-up analysis}

There were three transient gastric stenoses in the early postoperative period in the MiniMizer Extra group that resolved spontaneously during the first postoperative week. There were no early complications or early postoperative deaths. There was one early band penetration in the MiniMizer Extra group 6 months after the operation. The band was removed laparoscopically and this patient was excluded from further weight loss analysis. One patient died due to acute myocardial infarction 4 months after the LAGB operation in the SAGB group. One woman was excluded from weight loss analysis in a 1-year period because of pregnancy. Ten patients were unable to come for evaluation in the 1-year period after LAGB: 1 emigrated, 1 was after orthopaedic operation and 1 after trauma, and 7 were unable to come because of other reasons. All these ten patients were contacted by phone and weight loss was registered. Finally, 90 (41 SAGB and 49 MiniMizer Extra) patients arrived for 1-year follow-up evaluation, but 14 (9 SAGB and 5 MiniMizer Extra) of them refused the upper gastrointestinal tract endoscopy.

\section{Weight loss}

Weight loss parameters are shown in Table III. No statistically significant difference could be noted in either of the weight loss parameters. A significant difference in the proportion of patients who had achieved good or excellent weight loss results ( $\geq 50 \%$ of IEBMIL) was found in favour of the MiniMizer Extra group (29.6\% vs. 8.2\%, $p=0.006)$.

\section{Resolution of co-morbidities}

Changes in the frequency of co-morbidities are shown in Figure 4 . The most radical changes are noted in the frequency of gastric disorders $-87 \%$ of them disappeared or improved. Significant changes were also seen in the frequency of arterial hypertension, spinal and knee pain, type II diabetes mellitus, sleep apnoea, depression and elephantiasis - about a half of these co-morbidities improved or resolved. The frequency of metabolic syndrome decreased from $77.5 \%$ to $57.8 \%$. No significant differences were found between groups.

\section{Changes in quality of life}

The QoL improved from $0.02 \pm 1.2$ (range -3 to 2.7 ) to $0.894 \pm 1.1$ (range -2 to 3 ) points and though this is a significant improvement $(p<0.01)$, QoL is still stated as fair by M-A QoLQII. Changes in different aspects of QoL are shown in Figure 5. No significant differences were found between groups.

\section{Complications}

Oesophageal dilatation caused by over-inflation of the band was diagnosed by upper gastrointestinal tract radiography in one case in the MiniMizer Extra group. The band was deflated for a period of two months and oesophageal motility restored. One leakage was diagnosed from the ruptured connecting tube near the subcutaneous injection port in the MiniMizer Extra. The port was reconnected. Five cases $(9.3 \%)$ of band penetration were diagnosed endoscopically in the MiniMizer Extra group and no penetrations in the SAGB group ( $p=0.069)$. Four of these penetrations were asymptomatic. One of the pene- 
Table III. Weight loss parameters

\begin{tabular}{|c|c|c|c|c|}
\hline \multirow[t]{2}{*}{ Variable } & \multirow[t]{2}{*}{ All patients } & \multicolumn{2}{|c|}{ Comparative groups } & \multirow{2}{*}{$\begin{array}{c}\text { Difference } \\
\text { between } \\
\text { groups, } p\end{array}$} \\
\hline & & SAGB & MiniMizer Extra & \\
\hline IWL [kg] & $\begin{array}{l}19.5 \pm 11.9 \\
(-4.3-60)\end{array}$ & $\begin{array}{c}18.2 \pm 12 \\
(0-60)\end{array}$ & $\begin{array}{c}20.7 \pm 11.9 \\
(-4.3-54)\end{array}$ & 0.321 \\
\hline IBMIL [kg/m²] & $\begin{array}{c}6.7 \pm 3.9 \\
(-1.4-19.9)\end{array}$ & $\begin{array}{l}6.2 \pm 3.9 \\
(0-18.1)\end{array}$ & $\begin{array}{c}7.1 \pm 4.0 \\
(-1.4-19.9)\end{array}$ & 0.259 \\
\hline \%IWL [\%] & $\begin{array}{c}14.4 \pm 8.5 \\
(-2.6-37.9)\end{array}$ & $\begin{array}{c}12.9 \pm 8.1 \\
(0-35.4)\end{array}$ & $\begin{array}{c}15.7 \pm 8.7 \\
(-2.6-37.9)\end{array}$ & 0.112 \\
\hline$\%$ IBMIL [\%] & $\begin{array}{c}14.4 \pm 8.5 \\
(-2.6-37.9)\end{array}$ & $\begin{array}{c}12.9 \pm 8.1 \\
(0-35.4)\end{array}$ & $\begin{array}{c}15.7 \pm 8.7 \\
(-2.6-37.9)\end{array}$ & 0.112 \\
\hline IFML [kg] & $\begin{array}{c}12.3 \pm 7.6 \\
(-0.7-37.7)\end{array}$ & $\begin{array}{c}10.7 \pm 6.9 \\
(-0.4-31.4)\end{array}$ & $\begin{array}{c}13.5 \pm 7.9 \\
(-0.7-37.7)\end{array}$ & 0.090 \\
\hline IFMIL [kg/m²] & $\begin{array}{c}4.3 \pm 2.7 \\
(-0.2-14.7)\end{array}$ & $\begin{array}{c}3.7 \pm 2.4 \\
(-0.1-11.5)\end{array}$ & $\begin{array}{c}4.71 \pm 2.8 \\
(-0.2-14.7)\end{array}$ & 0.094 \\
\hline$\% I F M L[\%]$ & $\begin{array}{l}21.0 \pm 12.7 \\
(-1.2-58.0)\end{array}$ & $\begin{array}{c}18.1 \pm 11.9 \\
(-0.7-58.0)\end{array}$ & $\begin{array}{l}23.3 \pm 12.9 \\
(-1.2-56.1)\end{array}$ & 0.062 \\
\hline \%IFMIL [\%] & $\begin{array}{l}21.0 \pm 12.7 \\
(-1.2-58.0)\end{array}$ & $\begin{array}{c}18.1 \pm 11.9 \\
(-0.7-58.0)\end{array}$ & $\begin{array}{l}23.3 \pm 12.9 \\
(-1.2-56.1)\end{array}$ & 0.062 \\
\hline \%IEWL [\%] & $\begin{array}{c}33.1 \pm 21.9 \\
(-4.9-124.1)\end{array}$ & $\begin{array}{c}28.9 \pm 21.3 \\
(0-124.1)\end{array}$ & $\begin{array}{l}36.8 \pm 22.1 \\
(-4.9-91.1)\end{array}$ & 0.075 \\
\hline \%IEBMIL [\%] & $\begin{array}{c}33.1 \pm 21.9 \\
(-4.9-124.1)\end{array}$ & $\begin{array}{c}28.9 \pm 21.3 \\
(0-124.1)\end{array}$ & $\begin{array}{l}36.8 \pm 22.1 \\
(-4.9-91.1)\end{array}$ & 0.075 \\
\hline IFML/IWL & $\begin{array}{l}0.61 \pm 0.2 \\
(-0.1-1.2)\end{array}$ & $\begin{array}{l}0.6 \pm 0.2 \\
(-0.1-1.1)\end{array}$ & $\begin{array}{c}0.6 \pm 0.2 \\
(0-1.2)\end{array}$ & 0.364 \\
\hline
\end{tabular}

IWL - initial body weight loss, \%IWL - percentage of initial body weight loss, IBMIL - initial body mass index loss, \%IBMIL - percentage of initial body mass index loss, IFML - initial fat mass loss, \%IFML - percentage of initial fat mass loss, IFMIL - initial fat mass index loss, \%IFMIL - percentage of initial fat mass index loss, \%IEWL - percentage of initial excess weight loss, \%IEBMIL - percentage of initial excess body mass index loss, IFML/IWL - initial fat mass loss and initial body weight loss ratio (shows proportion of fat mass loss in body weight loss). Data are given as mean \pm SD (range)
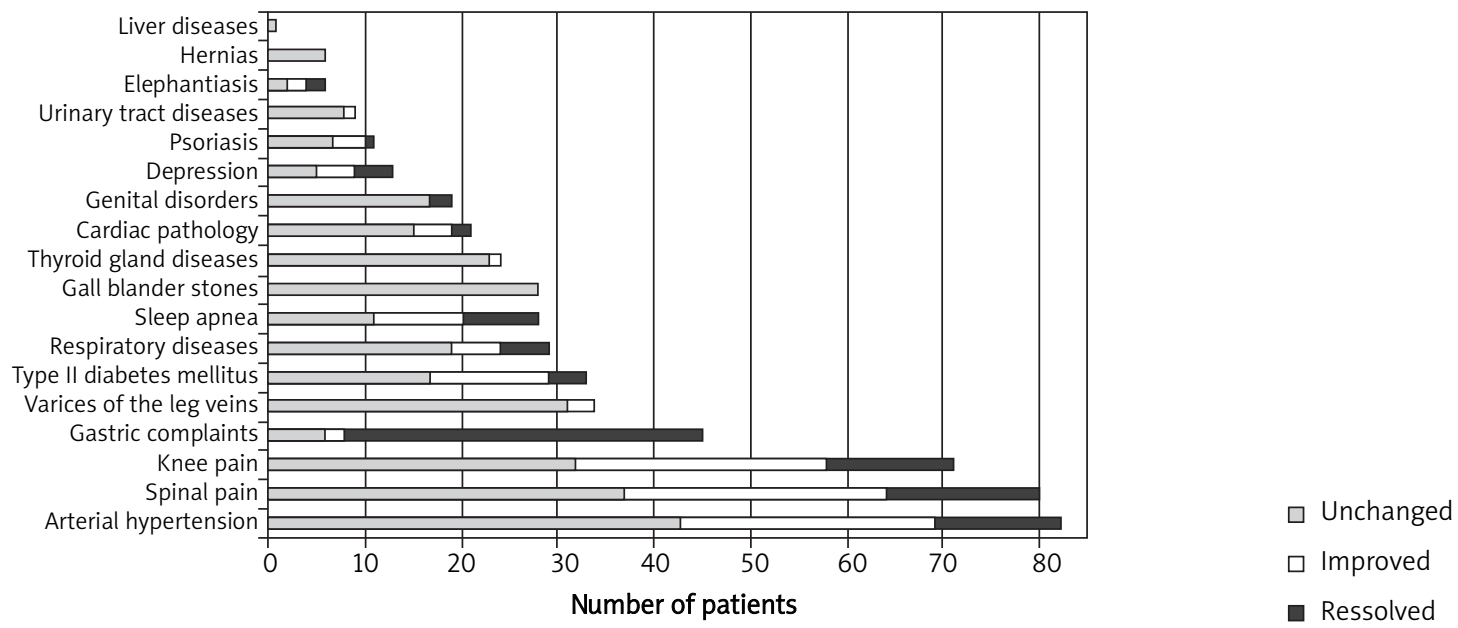

Figure 4. Changes in frequency of co-morbidities after 1 year (total width of the columns indicates initial frequency of the co-morbidities) 


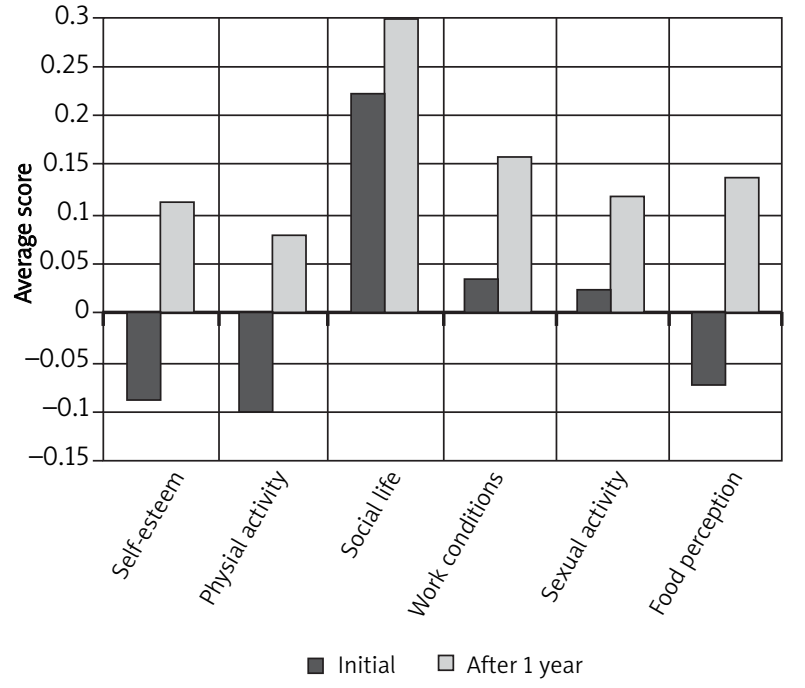

Figure 5. Changes in general self-esteem, activity levels and food perception by M-A QoLQII

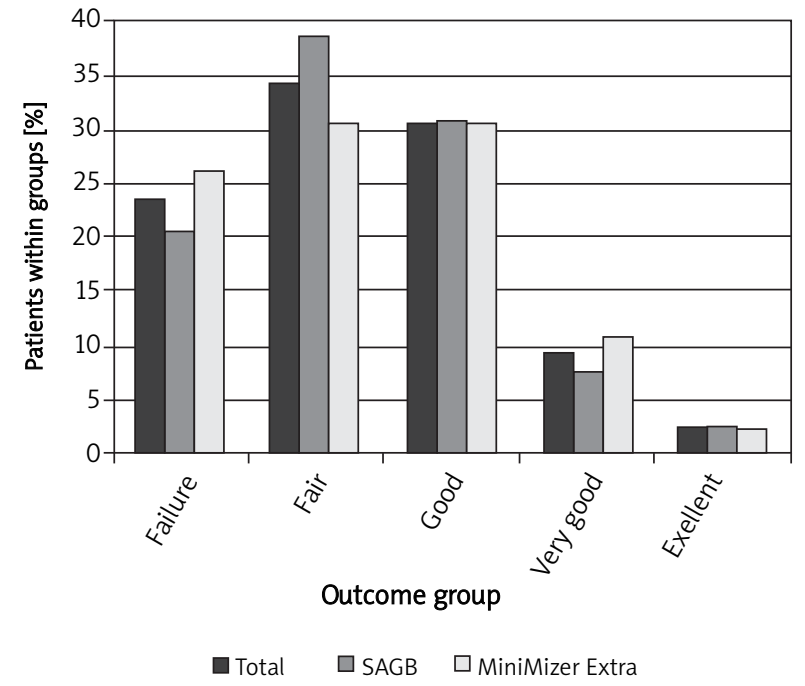

Figure 6. Distribution of patients in BAROS outcome groups trated bands was removed laparoscopically, while the other four patients were left under observation. As mentioned above, one death was registered in the SAGB group not associated with LAGB. No other complications were registered.

\section{Evaluation by BAROS}

The average BAROS score was $2.5 \pm 2.1$ in the SAGB group and $2.6 \pm 2$ in the MiniMizer Extra group $(p=0.811)$. The distribution of patients in BAROS outcome groups is shown in Figure 6. No significant differences were found between groups.

\section{Discussion}

The LAGB is usually reported as an effective bariatric procedure with a low perioperative complication rate and very low mortality [22, 25-30]. The mean excess weight loss is $45-65 \%$ after 2-5 years. The long-term complication rate varies in different studies. These include band slippage (or pouch dilatation) that occurs in $0.5-20 \%$ of patients, band penetration or migration of the band into the stomach lumen in $0.5-11 \%$ of patients, band or port infection in $1-3 \%$ of patients, and leaks either from the band, injection port or connecting tube in $1-25 \%$ of patients [22, 25-30]. Some of these complications are clearly associated with the operation method, for instance the frequency of band slippage dramatical- ly decreased after introduction of the pars flaccida technique instead of the previously used perigastric technique [21]. Leaks are more associated with the band itself, i.e. with the materials they are made of. An example is the Gastrobelt: Zieren et al. [31] found that 13 of 15 of these bands required removal because of leaks caused by band breakage (material failure). This problem is mostly eliminated with the use of quality materials, though the mechanical resistance of the silicone used for band manufacturing decreases in vivo after a few years [32]. Of course some of the leaks can occur while damaging the band, port or connecting tube during implantation or while accessing the port with the needle for band adjustment. The main and most serious complication usually leading to endoscopic or laparoscopic band removal is band penetration or migration through the gastric wall. The cause of this complication is still unclear. After introduction of the pars flaccida technique, the penetration rate decreased a little, suggesting the influence of the operation method [21]. Despite that, the band pressure on the gastric wall is believed to play the dominant role in penetration development. In this respect different bands are usually classified as low pressure-high volume or high pressure-low volume systems [5]. Unfortunately, this classification refers only to the volume and pressure within the balloon of the band, and the real pressure between the band and stomach wall in vivo has not 
been studied yet. Band fixation method is another factor that may be related to penetration. Most of the bands are secured to the anterior gastric wall by creating a gastric fold over the band using the socalled fixation with plication method. Now the MiniMizer Extra band, which is unique in this aspect, is available with a series of elastic retaining loops for direct fixation to the gastric wall with sutures. This band does not require folding of the anterior gastric wall (fixation without plication). Abundant literature on LAGB is available, mostly referring to SAGB and LAP-BAND devices. These two bands do not represent all possible differences in band design. Another problem is that most authors have used only one or the other band. Only a few series including two bands are available, and the follow-up is usually short [6-17]. This makes comparison between the two bands very difficult.

As far as we know, the present study is the first prospective randomised trial comparing the results of LAGB using the SAGB and MiniMizer Extra bands. We chose these bands as they have the most possible differences in their design. The time of the intraperitoneal part of the operation, i.e. band implantation time, is slightly shorter in the MiniMizer Extra group (47.3 vs. 56.2, $p=0.016$ ). This is possibly due to the easier fixation method of the MiniMizer Extra band in comparison with gastric fold formation with 4 sutures in the case of SAGB, but we do not think this time difference is of clinically significant importance. All weight loss parameters are slightly better in the MiniMizer Extra group, but a statistically significant difference was found only in the proportion of patients who reached $\geq 50 \%$ of excess body mass index loss (EBMIL) (29.6\% vs. $8.2 \%, p=0.006)$. There was no difference between groups in respect of resolution of co-morbidities or improvement of quality of life. Overall, there was no significant difference between groups regarding the incidence of early and long-term complications. The relatively high level of the band penetration rate in the MiniMizer Extra group should be mentioned the frequency of $9.3 \%$ (or even 11.1 valid \% in the endoscopically evaluated group) is very close to the upper limit of the published data but the difference between groups is not significant $(p=0.069)$. It should also be mentioned that four of five penetrations were totally asymptomatic and even more of these complications may be present in the group of patients who refused endoscopic evaluation.
This study has a number of limitations and the results in that respect must be interpreted with caution. Firstly, the short length of follow-up is far less than the minimum standard of 60 months considered as adequate to report the results of bariatric procedures. We will follow up these patients and will report 5-year results when they are available. Secondly, a group of lost follow-up patients, especially those who refused endoscopic evaluation, is also some kind of limitation.

In conclusion, no major significant differences were found between the two adjustable gastric bands. The early results comply with the published data and further results need to be confirmed by longer follow-up.

\section{References}

1. Kuzmak L. Silicone gastric banding: a simple and effective operation for morbid obesity. Contemp Surg 1986; 28: 13-8.

2. Hallberg D, Forsell P. Ballongband vid Behandling av Massiv Övervikt. Svensk Kirurgi 1985; 43: 106.

3. Belachew M, Legrand MJ, Vincent V, et al. Laparoscopic placement of adjustable silicone gastric banding in the treatment of morbid obesity: how to do it. Obes Surg 1995; 5: 66-70.

4. Buchwald H, Oien DM. Metabolic/bariatric surgery Worldwide 2008. Obes Surg 2009; 19: 1605-11.

5. Fried M, Lechner W, Kormanova K. Physical principles of available adjustable gastric bands: how they work. Obes Surg 2004; 14: 1118-22.

6. Benchetrit S, Combemalle F, De Villeneuve L, et al. Results of a retrospective comparative study between the Heliogast ${ }^{\circledR}$ and Lap-Band ${ }^{\circledR}$ rings in 850 patients. Obes Surg 2002; 12: 464-5.

7. Blanco-Engert R, Weiner S, Pomhoff I, et al. Outcome after laparoscopic adjustable gastric banding, using the Lap-Band ${ }^{\circledR}$ and the Heliogast ${ }^{\circledR}$ band: a prospective randomized study. Obes Surg 2003; 13: 776-9.

8. Collet D, Rault A, Sa Cunha A, et al. Laparoscopic adjustable gastric banding results after 2 years with two different band types. Obes Surg 2005; 15: 853-7.

9. Fabre JM, Nocca D, Lemoine MC, et al. Comparative study between lap-band and Swedish adjustable gastric banding. Obes Surg 2001; 11: 404

10. Gravante G, Araco A, Araco F, et al. Laparoscopic adjustable gastric bandings. A prospective randomized study of 400 operations performed with 2 different devices. Arch Surg 2007; 142: 958-61.

11. Hesse UJ, Berrevoet F, Ceelen W, et al. Das anpassbare Silicon Gastric Banding (ASGB, Bioenterics?) und das Schwedische anpassbare Gastric Banding (SAGB, Obtech?) zur Behandlung der morbiden Obesitas. Chirurg 2001; 72: 14-8.

12. Miller K, Hell E. Laparoscopic adjustable gastric banding: a prospective 4-year follow-up study. Obes Surg 1999; 9: 183-7.

13. Ponson AE, Janssen IM, Klinkenbijl JH. Laparoscopic adjustable gastric banding: a prospective comparison of two commonly used bands. Obes Surg 2002; 12: 579-82. 
14. Suter M, Giusti V, Worreth M, et al. Laparoscopic gastric banding. A prospective, randomized study comparing the lapband and the SAGB: early results. Ann Surg 2005; 241: 55-62.

15. Szewczyk T, Modzelewski B. Perioperative comparison of the MiniMizer ${ }^{\circledR}$ extra band with the other laparoscopic gastric bands. Obes Surg 2006; 16: 646-50.

16. Fried M, Kormanova K, Kasalicky M. Comparison of SAGB QC "Classic" Titanium Port and VelocityTM Port: pilot phase prospective randomized study on perioperative and short-term postoperative implantation outcomes. Obes Surg 2006; 16: 716-20.

17. Miller KA, Pump A. Mechanical versus suture fixation of the port in adjustable gastric banding procedures: a prospective randomized blinded study. Surg Endosc 2008; 22: 2478-84.

18. Kwiatkowski A, Paśnik K, Stanowski E, Badyda A. Regression of metabolic syndrome depending on type of bariatric surgery. Videosurgery and Other Miniinvasive Techniques 2009; 4: 53-8.

19. Oria HE, Moorehead MK. Updated Bariatric Analysis and Reporting Outcome System (BAROS). Surg Obes Relat Dis 2009; 5: 60-6.

20. Dadan J, Iwacewicz P, Hady HR. Quality of life evaluation after selected bariatric procedures using the bariatric analysis and reporting outcome system. Videosurgery and Other Miniinvasive Techniques 2010; 5: 93-99.

21. Di Lorenzo N, Furbetta F, Favretti F, et al. Laparoscopic adjustable gastric banding via pars flaccida versus perigastric positioning: technique, complications, and results in 2,549 patients. Surg Endosc 2010; 24: 1519-23.

22. Deitel M, Gagner M, Dixon JB, et al. Handbook of obesity surgery: current concepts and therapy of morbid obesity and related disease. FD-Communications. Toronto, Canada 2010.

23. Ballantyne GH. Measuring outcomes following bariatric surgery: weight loss parameters, improvement in co-morbid conditions, change in quality of life and patient satisfaction. Obes Surg 2003; 13: 954-64

24. Oria HE, Moorehead MK. Bariatric analysis and reporting outcome system (BAROS). Obes Surg 1998; 8: 487-99.

25. Brimas G. Chirurginis nutukimo gydymas. Ciklonas. Vilnius, Lithuania 2005.

26. Brimas G, Gaveliené E, Lipnickas V, et al. Nutukimo gydymas. Medicinos teorija ir praktika 2007; 2: 120-31.

27. Colquitt JL, Picot J, Loveman E, et al. Surgery for obesity. Cochrane Database Syst Rev 2009; CD003641.

28. Cunneen SA, Phillips E, Fielding G, et al. Studies of Swedish adjustable gastric band and lap-band: systematic review and meta-analysis. Surg Obes Relat Dis 2008; 4: 174-85.

29. Cunneen SA. Review of meta-analytic comparisons of bariatric surgery with a focus on laparoscopic adjustable gastric banding. Surg Obes Relat Dis 2008; 4: 47-55.

30. Fried M, Miller K, Kormanova K. Literature review of comparative studies of complications with Swedish band and Lap-Band. Obes Surg 2004; 14: 256-60.

31. Zieren J, Ablassmaier B, Enzweiler C, Müller JM. Disaster with a new type of band for gastric banding. Obes Surg 2000; 10: 22-5.

32. Lanthaler M, Schwienbacher F, Tembler J, et al. Mechanical resistance of gastric bands: comparison between new bands and bands within the patient for at least two years. Obes Surg 2004; 14: 524-7. 